\title{
Ethische Entscheidungsfindung in der Psychiatrie
}

\author{
Diana Meier-Allmendinger ${ }^{a}$, Julius Kurmann ${ }^{b}$, Ruth Baumann-Hölzlec \\ ${ }^{a}$ Dr. med. et lic. theol. Fachärztin für Psychiatrie und Psychotherapie, Leitende Ärztin Ambulatorium Klinik Schützen, Aarau, Team Stiftung Dialog Ethik \\ ${ }^{b}$ Dr. med., Luzerner Psychiatrie, Chefarzt Stationäre Dienste \\ ${ }^{c}$ Dr. theol., Institutsleitung der Stiftung Dialog Ethik
}

\begin{abstract}
Stimmt ein Patient dem vorgeschlagenen Behandlungsplan des Arztes oder der Ärztin nicht zu, entsteht das ethische Spannungsfeld zwischen Autonomieanspruch und Fürsorgeverpflichtung. In der Psychiatrie wird dies besonders deutlich. Erörtert werden die ethischen Hintergründe, Probleme und mögliche Umgangsformen anhand einer konkreten Patientensituation.
\end{abstract}

\begin{abstract}
Rechtlich werden auf der nationalen Ebene Handeln und Entscheiden in der Psychiatrie durch das Kindesund Erwachsenenschutzgesetz und kantonal durch die Patientengesetze geregelt. Dreh- und Angelpunkt der Gesetzgebung ist der Würde- und Autonomieanspruch des Individuums. Damit einher geht das Recht auf Leben und das Recht auf physische und psychische Integrität. Medizinische Handlungen stehen daher stets im Spannungsfeld zwischen der Verpflichtung zur Lebenserhaltung (Fürsorgeverpflichtung) und dem Respekt gegenüber dem Integritätsanspruch (Autonomieanspruch) des Patienten.
\end{abstract}

\section{Prise de décision éthique en psychiatrie}

En psychiatrie, comme en médecine et dans les soins en général, la volonté du patient forme le pilier de toute prise de décision éthique. Lorsque celle-ci n'est pas connue ou qu'elle est altérée par une maladie psychique, la situation peut devenir un réel dilemme sur le plan éthique. A l'aide d'un exemple concret, le présent article met en évidence les différentes facettes du champ de tension éthique entre autonomie du patient et obligation de prêter assistance de l'équipe soignante. Une situation complexe à laquelle viennent encore s'ajouter les tensions entre l'exigence éthique d'autonomie du patient et les capacités réelles d'autonomie de celui-ci, ou entre l'obligation éthique de prêter assistance de l'équipe soignante et les possibilités concrètes dont elle dispose pour ce faire. Or de telles situations nécessitent souvent beaucoup de temps et d'énergie de la part de l'équipe soignante. A cet égard, les procédures de décision multiprofessionnelles, comme le dialogue en 7 étapes ( 7 -Schritte-Dialog»), peuvent se révéler être d'une grande aide pour les personnes impliquées et contribuent ainsi à économiser les ressources. Ces procédures ne sont toutefois efficaces que dans des organisations qui connaissent une culture éthique vivante, et en ce sens, l'éthique même de l'organisation et l'éthique dans I'organisation se complètent mutuellement.
In der konkreten Patientensituation wird das Verhältnis zwischen Fürsorgeverpflichtung und Autonomieanspruch durch die Urteilsfähigkeit des Patienten bestimmt. Der Anspruch auf Integrität wird beim Urteilsfähigen höher gewichtet als das Recht auf Leben. Deshalb gilt jede medizinische Handlung als Körperverletzung, in die der Patient einwilligen muss. Dadurch erhält der Urteilsfähige indirekt die Freiheit zur Selbstschädigung, denn solange er urteilsfähig ist, kann ihn aufgrund seines Abwehrrechtes niemand zu einer Behandlung zwingen. Dieses Abwehrrecht kann ein urteilsfähiger Patient sogar für die Situation der Urteilsunfähigkeit wahrnehmen, indem er in einer Patientenverfügung im Voraus den Behandlungsrahmen festlegt.

Ist aber der Wille eines Menschen nicht bekannt oder die Willensbildung durch eine psychische Erkrankung beeinträchtigt, hat das Recht auf Leben Vorrang gegenüber dem Integritätsanspruch, d.h., die Fürsorgeverpflichtung erhält stärkeres Gewicht.

Während in der somatischen Medizin nicht mehr der behandelnde Arzt oder die Ärztin bei Urteilsunfähigkeit die Entscheidungsverantwortung trägt, sondern die im Gesetz festgelegten Stellvertreter, entscheidet über psychisch kranke und urteilsunfähige Menschen in der Psychiatrie nach wie vor der behandelnde Arzt oder Ärztin gemäss den Regeln der Fürsorgerischen Unterbringung. Die Freiheit zur Selbstschädigung, die somatisch kranken Menschen zugestanden wird, erlischt bei schwer verwahrlosten oder psychisch kranken Menschen. Sie dürfen auch gegen ihren Willen behandelt werden. Damit wird der ethische Anspruch auf Würde und Autonomie der psychisch Kranken jedoch nicht aufgehoben. Auch sie sind gemäss 


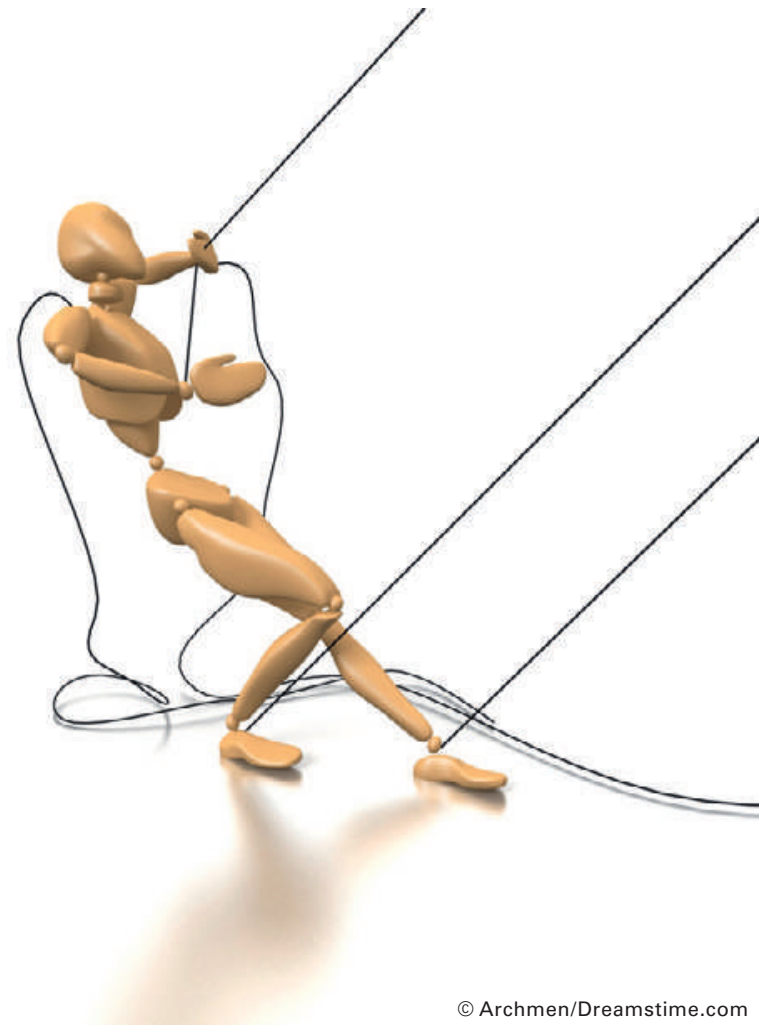

Eine Grundsatzfrage: Ist der Patient autonom und urteilsfähig oder ein Gefangener seiner psychischen Krankheit?

ihrem mutmasslichen nicht beeinträchtigten Willen und im Sinne ihrer Interessen zu behandeln und $\mathrm{zu}$ betreuen, die z.B. in einer Patientenverfügung festgelegt sind. Für die Behandlung und Betreuung in der Psychiatrie ist gemeinsam mit dem Patienten und falls vorhanden - mit seiner Vertrauensperson ein individueller Behandlungsplan zu erstellen.

Das Recht überlässt die Bestimmung des individuellen Behandlungsmasses in Form des Behandlungsplans dem Behandlungsteam. In den meisten Patientensituationen kann bei der Erstellung des Behandlungsplans auf die Regeln der ärztlichen Kunst des psychiatrischen Fachwissens zurückgegriffen werden. In Situationen aber, in denen die Behandlung unklar oder strittig und das Handeln nicht selbstverständlich ist, kommt die ethische Güterabwägung ins Spiel.

\section{Spannungsfelder sichtbar machen}

In einer konkreten Patientensituation ist es Aufgabe der Ethik, die individuelle Behandlung und Betreuung im Rahmen einer transparenten und verbindlichen Güterabwägung zu bestimmen. Diese bewegt sich in zwei Spannungsfeldern: Erstens im horizontalen Spannungsfeld zwischen Autonomieanspruch und Fürsorgeverpflichtung. Zweitens im vertikalen Spannungsfeld zwischen eben diesem Autonomieanspruch und der
Fürsorgeverpflichtung auf der normativen Ebene, die nun ihrerseits beide in einem Spannungsverhältnis sowohl zu den konkreten stets beschränkten Autonomiefähigkeiten des Patienten als auch den beschränkten Behandlungs- und Betreuungsmöglichkeiten des Behandlungsteams andererseits auf der empirischen Ebene stehen.

\section{Konkrete Beispiele der Spannungsfelder}

Die gängige medizin-ethische Entscheidungsfindung orientiert sich an den bekannten Prinzipien "Autonomie», "Nicht schaden», "Gutes tun» und "Gerechtigkeit». Dabei kommt dem Autonomieprinzip eine übergeordnete Funktion zu: Patienten sollen selbstbestimmt einer medizinischen Behandlung zustimmen oder diese ablehnen können. Aus der Perspektive des Behandlungsteams erhält jedoch das Prinzip Fürsorge ein besonderes Gewicht, wenn die Ablehnung einer Behandlung für das behandelnde Team nicht nachvollziehbar ist. Daraus entsteht ein Spannungsfeld zwischen den Patientenwünschen und der Fürsorge (-pflicht) der Behandler, das nicht selten in der Psychiatrie zu einer Dilemmasituation führen kann.

Ein Beispiel: Herr M. leidet seit Jahren unter einer rezidivierenden Depression mit gesunden und symptomfreien Intervallen. Er erlebt gute Zeiten mit seiner Ehefrau und seiner Familie, kann gut arbeiten und sich um die Kinder kümmern. Er nimmt die Medikamente auch während der symptomfreien Phasen gewissenhaft ein. Nach fünf Jahren ohne depressive Symptomatik stellen sich erneut Symptome einer Depression ein mit Gefühlen der Insuffizienz, der Wertlosigkeit, der Freudlosigkeit und Antriebslosigkeit. Aus diesem Grund rät ihm der zuständige Psychiater zu einer teilstationären (Tagesklinik) oder stationären Behandlung. Der Patient kann sich zu einer teil- bzw. stationären Behandlung nicht entschliessen. Er ist nicht suizidal. Der Therapeut versucht ihn zum Eintritt zu motivieren, da er der Überzeugung ist, Herr M. könnte rascher gesunden und würde im Umgang mit seiner Depression dazulernen. Herr M. lehnt ab. Aufgrund fehlender Selbstgefährdung besteht rechtlich kein Grund, ihn in eine Klinik einzuweisen. Unter therapeutischer Sicht jedoch gäbe es mehrere Gründe: Die ambulanten Therapiemöglichkeiten sind ausgeschöpft, eine intensivere Therapie ist therapeutisch indiziert. Der Psychiater befindet sich im Dilemma zwischen der Akzeptanz des Willens des Patienten (Autonomie) und seiner Überzeugung, durch eine weiterführende Behandlung des Patienten Gutes zu tun (Fürsorge). Damit öffnet sich das Spannungsfeld zwischen den rechtlichen und ethischen Grundnormen der Autonomie, die jedem Patien- 
ten zustehen und als Norm zu akzeptieren sind, und den tatsächlich vorhandenen Fähigkeiten des Patienten, auch frei und unabhängig entscheiden zu können. Diese Ambivalenz ist ein bekanntes Symptom der Depression und zeichnet sich dadurch aus, dass eine Entscheidung zwischen zwei Möglichkeiten nicht getroffen werden kann. Durch die Depression ist seine Fähigkeit zur freien Entscheidung deutlich beeinträchtigt.

In der Praxis ist es wichtig, dass sich Therapeut und Patient über die genannten Spannungsfelder bewusst werden. Die Orientierung an diesen Prinzipien steckt den Rahmen ab. Eine gute Lösung im konkreten Fall entsteht aber nur, wenn die vorhandenen Fähigkeiten des Patienten gemeinsam erörtert werden.

Nicht nur bei Krankheiten mit deutlicher Beeinträchtigung des Realitätsbezuges wie in manischen, psychotischen oder schwer depressiv-suizidalen Zuständen kann die Entscheidungsfähigkeit oder die Willensbildung grundsätzlich beeinträchtigt sein. In vielen Krankheitsbildern ist die Willensbildung durch die Erkrankung betroffen: Die emotional instabile Borderline-Patientin, die ihren Schneideimpuls nicht mehr

\section{Ist die Willensbildung durch eine psychische Erkrankung beeinträchtigt, hat das Recht auf Leben Vorrang gegenüber dem Integritäts- anspruch.}

verhindern kann; der Suchtkranke, der seinen Willen in Bezug auf Alkoholkonsum nicht unter Kontrolle hat; Patienten mit einer Zwangsstörung, in denen die Zwänge stärker sind als der Wille, eine Zwangshandlung nicht durchzuführen; Personen, die aufgrund einer Störung ihrer Persönlichkeit ihre Impulse in bestimmten Situationen nicht mehr kontrollieren können und dadurch andere verbal oder körperlich verletzen. Dies sind Beispiele für beeinträchtigte Fähigkeiten zur freien Entscheidung, da jede Willensbildung an eine bestimmte Person und ihre dafür vorhandenen Fähigkeiten gebunden ist. Gleichwohl darf diesen Personen aufgrund ihrer Diagnose ihre Urteilsfähigkeit oder ihre Autonomie nicht grundsätzlich abgesprochen werden, dies gilt auch für die Freiheit ihres Willens. Für die Behandler und das therapeutische Vorgehen können sich daraus mannigfaltige Konfliktsituationen ergeben.

Die konkrete Entscheidungsfindung kann nur innerhalb der therapeutischen Beziehungen und im Prozess als ethischer Dialog der Beteiligten gelingen. Ein ethischer Dialog ist nicht nur ein interprofessioneller Austausch über Fakten, sondern zielt darauf ab, über eine ethische Güterabwägung das angemessene Handeln in einer individuellen Situation zu bestimmen. Ein ethischer Dialog hat sowohl inhaltliche als auch formale Voraussetzungen. Im Folgenden vollziehen wir anhand einer konkreten Patientensituation beispielhaft einen ethischen Güterabwägungsprozess mit dem «7-Schritte-Dialog». Dieses Entscheidungsfindungsmodell wurde bereits mehrfach ausgewertet und wird auch in der Psychiatrie in schwierigen Entscheidungssituationen angewendet.

\section{Patientensituation - Verweigerung der somatischen Therapie und/oder Nahrungsverweigerung}

Eine 60-jährige Patientin leidet unter einer wahnhaften Störung. Sie ist nicht bereit, die dringend notwendige somatische Medikation (Antikoagulation) einzunehmen. Unter Druck nimmt sie Neuroleptika ein. Die neuroleptische Behandlung verbessert aber das Zustandsbild nicht. Zusätzlich verweigert sie die Aufnahme der Nahrung und das Trinken.

Es stellt sich die Frage, ob dieser Entscheid der Patientin akzeptiert werden soll und kann oder ob eine zwangsmässige Applikation der somatischen Medikation notwendig wird.

\section{Schritt: Ethisches Dilemma}

Lebenserhaltung versus Respekt der Patientenautonomie im Sinne des Abwehrrechtes?

Kann die Aussage der Patientin wirklich als eigenständiger Patientenwille verstanden werden oder ist dieser Patientenwille durch das psychotische Erleben beeinträchtigt? Darf eine ärztliche Handlung unterlassen werden im Wissen, dass die Unterlassung potentiell gefährlich ist, ja sogar den Tod bringen kann? Unser gemeinsames Problem mit dieser Patientin und ihrem Vergiftungswahn ist, dass wir nicht wissen, ob sie in Bezug auf die Notwendigkeit der Einnahme der somatischen Medikation urteilsfähig ist (d.h. wir ihre Medikamentenverweigerung akzeptieren müssen) oder ob die Verweigerung der Einnahme der somatischen Massnahme Teil ihrer psychischen Erkrankung ist und wir gegen den Willen die Patientin trotzdem somatisch behandeln müssen.

\section{Schritt: Kontextanalyse des ethischen Dilemmas} Grundsätzlich besteht bei jedem urteilsfähigen, somatisch kranken Patienten eine Freiheit zum selbstschädigenden Verhalten. Kann dieser geäusserte Patientenwille bei unserer Patientin als eigenständige, freie Entscheidung verstanden werden? In unserer Situation haben wir mit der Antikoagulation ein effektives und erprobtes Mittel der Behand- 
lung. Es kann die Thrombosenbildung und allenfalls spätere Emboliegefahr deutlich mindern.

Im Gegensatz zur psychiatrischen Behandlung gegen den Willen der Patienten haben wir im somatischen Bereich bezüglich Zwangsbehandlungen eine ungenügende Rechtsgrundlage.

\section{Dieses Entscheidungsfindungsmodell wurde} mehrfach ausgewertet und auch in der Psychiatrie angewendet.

Die Entscheidungskaskade im Kindes- und Erwachsenenschutzgesetz gilt auch für somatische Therapieentscheide für psychisch Kranke. Aber: In der Psychiatrie hat der Arzt die Möglichkeit, Patienten gegen ihren Willen zu behandeln, falls die Behandlungsverweigerung aufgrund der psychischen Erkrankung erfolgt.

Die vorliegende Patientensituation ist daher rechtlich unklar.

\section{Schritt: Werteanalyse des ethischen Dilemmas}

Die Urteilsfähigkeit misst sich an dem zu entscheidenden Gegenstand. Wir müssen also klären, ob die Patientin bezüglich der Verweigerung der Medikamenteneinnahme urteilsfähig ist.

Juristisch verstehen wir unter der Urteilsfähigkeit Folgendes:

- Fähigkeit, die gegebene Situation zu erfassen;

- Fähigkeit, die gegebenen Informationen zu verstehen;

- Fähigkeit, die voraussehbaren Konsequenzen einzuschätzen;

- Fähigkeit, die voraussehbaren Konsequenzen zu werten;

- Fähigkeit, eine Wahl zu treffen, diese zu äussern und gemäss dieser zu handeln.

Wir beurteilen die Urteilsfähigkeit der Patientin bezüglich der Entscheidung, die Medikamente nicht einzunehmen. Wir stellen fest, dass die Patientin eine Medikation nicht per se in Frage stellt. Sie hat auch explizit ihren Lebenswillen formuliert. Vielmehr glaubt sie, dass die Behandler sie mit den Medikamenten vergiften wollen. Medikamente von zu Hause würde sie einnehmen. Somit ist in diesem Fall keine Urteilsfähigkeit gegeben.

Wenn wir davon ausgehen, dass keine Urteilsfähigkeit bezüglich des entscheidenden Gegenstandes vorhan- den ist, stehen sich die Prinzipien "Gutes tun" und «Schaden» gegenüber. Da die Patientin nach menschlichem Ermessen nicht in absehbarer Zeit mit einer Behandlung sterben würde, muss der Behandler das Unterlassen einer medizinischen Behandlung rechtfertigen. Ihr Tod darf aufgrund ihrer Ablehnung nicht in Kauf genommen werden.

\section{Schritt: Entwurf von mindestens drei Handlungsmöglichkeiten}

Wir können folgende hierarchische Vorgehensweisen vorschlagen:

1. Medikamentenabgabe durch eine Person, zu der die Patientin Vertrauen hat;

2. Einsatz von einer Peer-Mitarbeiterin;

3. Einbezug von Angehörigen;

4. Psychiatrische Behandlung auch gegen den Willen der Patientin;

5. Anrufen der KESB;

6. Behandlung auch mit der somatischen Medikation gegen den Willen der Patientin,

7. Unterlassung einer Behandlung.

Zusammen mit dem Behandlungsteam werden in der nächsten Phase die Schritte 5 bis 7 diskutiert und erarbeitet. Diese sind:

- Gemeinsame Analyse der Handlungsmöglichkeiten (5. Schritt);

- Konsensfindungsprozess und Handlungsentscheid (6. Schritt)

- Kommunikation und Überprüfung des Handlungsentscheids (7. Schritt).

Das Behandlungsteam entschied sich, gemäss der Handlungskaskade vorzugehen.

Wie die obige Falldarstellung und Diskussion zeigt, ist ein ethischer Dialog eine breit abgestützte, moderierte Diskussion innerhalb des gesamten Behandlungsteams. Dieser multiprofessionelle Prozess ist wichtig, damit sich alle beteiligten Berufsgruppen angesprochen fühlen und den Entscheid mittragen und umsetzen können. Dieses Vorgehen setzt in einer Organisation eine Kultur bewusster ethischer Reflexion beim alltäglichen Entscheiden und Handeln auf allen Verantwortungsebenen voraus. Ethik in einer Organisation beschränkt sich daher keinesfalls auf Fallbesprechungen, sondern befasst sich breit mit der jeweiligen Hauskultur und der Ethik der Organisation. 